\title{
QUANTIZATION OF LINEAR POISSON STRUCTURES AND DEGREES OF MAPS
}

\author{
MICHAEL POLYAK
}

\begin{abstract}
Kontsevich's formula for a deformation quantization of Poisson structures involves a Feynman series of graphs, with the weights given by some complicated integrals (using certain pullbacks of the standard angle form on a circle). We explain the geometric meaning of this series as degrees of maps of some grand configuration spaces; the associativity proof is also interpreted in purely homological terms. An interpretation in terms of degrees of maps shows that any other 1 -form on the circle also leads to a $*$-product and allows one to compare these products.
\end{abstract}

\section{INTRODUCTION}

1.1. Kontsevich's quantization. Recently, Kontsevich [4 provided a deformation quantization of the algebra of functions on an arbitrary Poisson manifold. The key ingredient of his construction is the quantization formula for $\mathbb{R}^{d}$, which is then extended to the case of general manifolds using formal geometry. The terms of the star product are identified with certain Feynman graphs, and their coefficients ("weights") are given by some complicated integrals (involving certain pullbacks of the standard volume form on a torus over the corresponding configuration spaces).

The first non-trivial case of Kontsevich's construction is for a linear Poisson brackets, i.e. Poisson structures on a dual of a Lie algebra. In this paper we consider only this case (see Section 1.3 for a discussion).

Removal of the two external vertices splits any graph appearing in the definition of the star product into several connected components. The weight of a graph is the product of weights of its connected components. In a linear case each of them may have at most one cycle, which should consist of oriented edges. It turns out that one may restrict the class of graphs in Kontsevich's construction to trees, excluding graphs with a non-trivial first homology. The weights of these tree graphs coincide with the coefficients of the usual Gutt's star product [2] arising from the Campbell-Baker-Hausdorff formula 3. Thus Kontsevich's quantization differs from the $\mathrm{CBH}$-formula essentially only by the contributions of graphs with one cycle, in particular of the so-called "wheels" (see Section 4.6).

1.2. Main results and the organization of the paper. An important problem related to Kontsevich's construction for $R^{d}$ is to interpret geometrically, and show a way to compute, the weights of graphs. In some partial cases such computations

2000 Mathematics Subject Classification. Primary: 53D55; Secondary: 55R80, 57R35.

Key words and phrases. Deformation quantization, Poisson structures, Feynman graphs, configuration spaces, compactification.

Partially supported by the Israeli Science Foundation grant 86/01 and the Technion grant for promotion of research. 
were done in 3. However, these direct computations are complicated and not illuminating. One of the reasons for a lack of a geometrical interpretation of these integrals seems to be that some special properties of the standard angle form on $S^{1}$ are significantly involved in Kontsevich's proofs. Thus two natural questions are whether other 1-forms on $S^{1}$ also lead to an associative star product, and if yes, how do these products depend on the choice of a form. We answer both questions in the linear case.

First we show than any 1-form on $S^{1}$ gives an associative star product. This is done by an interpretation of the associativity as a vanishing of some cocycle in the relative top cohomology of a grand configuration space $C_{n, 3}$. These spaces are obtained by gluing together the configuration spaces corresponding to graphs along their boundary strata. These boundary strata are of two types: principal and hidden. The gluings along the principal strata are done according to the Jacobi relation. Hidden strata are zipped-up, i.e. glued to itself by some involutions. By choosing singular forms concentrated in one point on a torus, one gets star products with rational coefficients (with a straightforward estimate on the denominators). These coefficients can be computed by a simple combinatorial count.

We then turn to a problem of comparison of these star products. This is done in a similar fashion, by identifying terms in these star products with some fixed top cohomology class of a similar grand configuration space $C_{n, 2}$. This identification involves, however, a capping of some additional- external- boundary strata by the configuration spaces of the wheels. Thus products for different differential forms on $S^{1}$ may differ only by the weights of the wheels. We show that the tree (i.e. 0-loop) part of all star products coincide and are equal to Gutt's star product. We also show that any differential form supported on a semi-circle leads to Gutt's product.

1.3. Related problems. The point of view of this paper was heavily motivated by a similar interpretation of the Feynman series appearing in the perturbative Chern-Simons theory and used to produce a universal invariants of knots and 3manifolds, see e.g. 1 6 6]. The construction of the star product considered in this note is quite similar (in particular, it is interesting that a similar antisymmetry and Jacobi relations appear in the gluing construction), although there are of course few differences (in the Chern-Simons theory the edges are not oriented, all vertices are trivalent- which implies a simple involution argument showing the vanishing of the hidden strata- and the spaces after gluing are closed). There is a clear common denominator in these constructions; in fact, this note was initiated as a check that the same technique may be applied. I believe that this technique of producing grand configuration spaces by gluings is quite powerful; it would be quite interesting to find some other applications.

While I strongly believe that the approach used in this paper can be applied for general Poisson structures, the zip-up argument of Section 3.5 for hidden faces then works only up to degree six, so a new argument is needed to prove a conjecture about vanishing of the weights of the hidden strata. When this paper was already finished, the author found a modification of this construction which works for quadratic Poisson structures (to be discussed in a forthcoming paper). The general case, however, still remains open. 


\section{GRAPHS AND DIFFERENTIAL OPERATORS}

2.1. Admissible graphs. Througout the paper we will deal with graphs $G$ such that

- the edges of $G$ are oriented;

- the set of vertices of $G$ is subdivided into two disjoint subsets: the internal vertices $V^{i}$ and the external vertices $V^{e}$;

- the set of external vertices is ordered;

- $G$ does not contain double or looped edges.

An orientation of $G$ is an ordering of outgoing edges in each vertex, up to a negation in any two vertices. Following Kontsevich [4, we will call an oriented graph $G$ admissible if

- exactly two edges start in each internal vertex, and no edges start in any external vertex.

Since we will deal primarily with the linear case, i.e. the Poisson brackets on a dual of a Lie algebra, we define a Lie-admissible graph as an (oriented) admissible graph such that

- in each internal vertex there ends at most one edge.

Note that this condition implies that each connected component of a graph obtained from $G$ by a removal of all external vertices is either a tree, or contains one cycle, which consists of oriented edges.

Denote by $\mathcal{G}_{n, m}$ the set of all Lie-admissible graphs with $n$ internal and $m$ external vertices. For example, $\mathcal{G}_{0,2}$ is generated by the graph with two (external) vertices and no edges. The "wedge" graph with one internal vertex connected to two external vertices by a pair of edges (see the graph $G^{\prime}$ of Figure 1), gives two generators of $\mathcal{G}_{1,2}$, which differ by the ordering of the edges. Denote $\mathcal{G}_{m}=\amalg_{n} \mathcal{G}_{n, m}$.

Note that the orientation of a graph with a set $E$ of edges induces an orientation of $\mathbb{R}^{|E|}=R^{2} \times \ldots \mathbb{R}^{2}$ as in [4: choose an arbitrary ordering of all internal vertices and then take ordered pairs of edges starting at 1-st, 2-nd, etc., vertex. For technical reasons (namely, the Jacobi relation (3) for labelled graphs) we will often need to consider more general orderings of $E$. A labelled graph is an admissible graph endowed with an arbitrary ordering of its edges, compatible with the above orientation of $R^{|E|}$.

Remark 2.1. Note that our labellings are more general than in [4]: we consider all orderings of edges, so for a non-oriented graph with $n$ internal vertices and no nontrivial automorphisms the number of labellings is $(2 n)$ !. Picking an orientation of $G$ cuts this number in half. In general we should also take into account the number of automorphisms of $G$ (preserving the order of external vertices), so the number of different labellings of an oriented graph is $\mid$ Aut $\left.G\right|^{-1}(2 n) ! / 2$.

Denote by $\widetilde{\mathcal{G}}_{n, m}$ the set of all labelled Lie-admissible graphs with $n$ internal and $m$ external vertices, and set $\mathcal{G}_{m}=\amalg_{n} \mathcal{G}_{n, m}$. There is an obvious forgetful map $\widetilde{\mathcal{G}}_{n, m} \rightarrow \mathcal{G}_{n, m}$; abusing notation, we will denote the image of $G$ also by $G$.

2.2. Product and composition of graphs. There are several important operations on graphs.

Firstly, there is a product $\mathcal{G}_{n, m} \times \mathcal{G}_{n^{\prime}, m} \rightarrow \mathcal{G}_{n+n^{\prime}, m}$ : we define $G \cdot G^{\prime}$ to be the graph obtained by juxtaposition of $G$ and $G^{\prime}$, followed by the identification of their 
external vertices. See Figure 1 . This makes $\mathcal{G}_{m}$ into a semigroup. In particular, $\mathcal{G}_{1}$ is generated by $n$-spiked "wheels", i.e. by graphs with $n$ interior vertices forming an oriented cycle of edges and connected to the exterior vertex by $n$ spikes, see Figure 9a.

Secondly, there is a composition $\circ_{i}: \mathcal{G}_{n, m} \times \mathcal{G}_{n^{\prime}, m^{\prime}} \rightarrow \mathcal{G}_{n+n^{\prime}, m+m^{\prime}-1}, i=1, \ldots, m$ of two admissible graphs $G \in \mathcal{G}_{n, m}, G^{\prime} \in \mathcal{G}_{n^{\prime}, m^{\prime}}$ defined by inserting $G^{\prime}$ in $i$-th external vertex of $G$. This insertion is done in the following way. Cut out a small disc surrounding the $i$-th external vertex $v_{i}$ of $G$ and place there a small copy of $G^{\prime}$. Now, sum over all possible ways of attaching the incoming edges of $v_{i}$ to the vertices of $G^{\prime}$, which result in a Lie-admissible graph. See Figure 1 $b$. The external vertices of $G \circ_{i} G^{\prime}$ are ordered in a natural way: $\left\{1, \ldots, i-1,1^{\prime}, \ldots, m^{\prime}, i+1, m\right\}$.

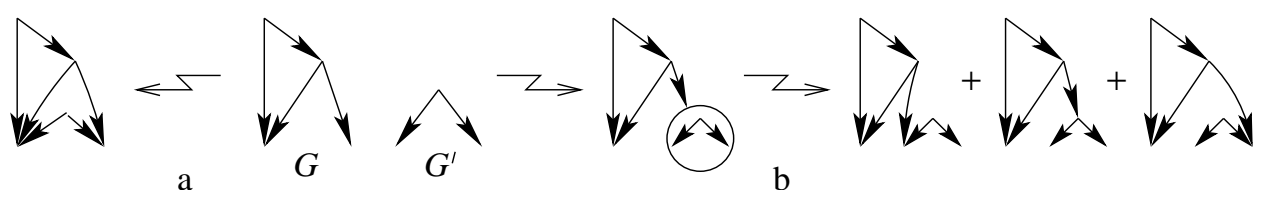

Figure 1. A product $G \cdot G^{\prime}$ and a composition $G \circ_{2} G^{\prime}$ of graphs

We may combine all $\circ_{i}$ 's into one composition on the vector space $\mathbb{Q G}$ over $\mathbb{Q}$ spanned by $\mathcal{G}$ :

$$
G \circ G^{\prime}=\sum_{i=1}^{m}(-1)^{i} G \circ_{i} G^{\prime}
$$

While non-associative, this operation defines a pre-Lie structure on $\mathbb{Q} \mathcal{G}$, i.e., for any $x, y, z \in \mathcal{G}$ we have $x \circ(y \circ z)-(x \circ y) \circ z=x \circ(z \circ y)-(x \circ z) \circ y$. Hence the bracket $\left[G, G^{\prime}\right]=G \circ G^{\prime}-G^{\prime} \circ G$ defines a Lie algebra structure on $\mathbb{Q} \mathcal{G}$.

In a similar way, one may define a co-product on $\mathbb{Q} \mathcal{G}$ by $\Delta G=\sum_{G^{\prime} \subset G}\left(G / G^{\prime}\right) \otimes$ $G^{\prime}$, where the summation is over all Lie-admissible subgraphs $G^{\prime}$ of $G$ s.t. the quotient graph $G / G^{\prime}$ (obtained by shrinking $G^{\prime}$ into a point) is also Lie-admissible.

All above operations may be also defined on labelled graphs in an obvious way.

2.3. Differential operators assigned to graphs. Let $\alpha$ be a bi-vector field on an open domain $\mathcal{U}$ in $\mathbb{R}^{d}$. In the coordinates $x^{i}, i=1,2, \ldots, d$ on $R^{d}$ it is given by $\alpha=\sum_{i, j} \alpha^{i j}(x) \partial_{i} \wedge \partial_{j}$, where $\partial_{i}=\frac{\partial}{\partial x^{i}}$. To each admissible graph $G \in \mathcal{G}_{n, m}$ we assign a polydifferential operator $B_{G}:\left(C^{\infty}(\mathcal{U})\right)^{\times m} \rightarrow C^{\infty}(\mathcal{U})$ as in [4. Namely, put $\alpha^{i(v) j(v)}$ at each internal vertex $v$ of $G$ and put the corresponding derivatives $\partial_{i(v)}$ and $\partial_{j(v)}$ at two edges starting at $v$. Thus we have a derivative $\partial_{e}$ assigned to each edge of $G$, and a tensor $\alpha^{i j}$ at each internal vertex. We will call such an assignment a state of $G$. Finally, put a function $f_{i}$ at $i$-th external vertex, $i=1, \ldots, m$. The operator $B_{G}$ is defined by the following formula:

$$
B_{G, \alpha}\left(f_{1}, \ldots, f_{m}\right)=\sum_{\text {states }}\left(\prod_{v \in V^{i}}\left(\prod_{e \in E(v)} \partial_{e}\right) \alpha^{i(v) j(v)}\right) \times\left(\prod_{v \in V^{e}}\left(\prod_{e \in E(v)} \partial_{e}\right) f_{v}\right)
$$


where $E(v)$ denotes the set of incoming edges of a vertex $v$. For example, for the graph $G$ in the left hand side of Figure 1 we have

$$
B_{G}(f, g)=\sum_{i, j, k, l} \alpha^{i j} \partial_{j}\left(\alpha^{k l}\right) \partial_{i} \partial_{k}(f) \partial_{l}(g) .
$$

If $\alpha$ is linear, its second derivatives vanish, so $B_{G}=0$ for any graph $G$ which contains a vertex with at least two incoming edges. Thus in the linear case it suffices to consider only Lie-admissible graphs.

2.4. Antisymmetry and Jacobi relations on graphs. We put $G=-G^{\prime}$ if two admissible graphs $G, G^{\prime}$ differ only by the ordering of two edges starting in some internal vertex. Here is a graphical version of this antisymmetry relation $(A S)$ :

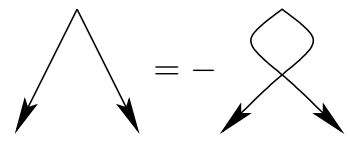

Here and further on, in figures at each internal vertex we use the ordering of two outgoing edges induced by the orientation of the plane, unless specified otherwise.

We impose the following Jacobi relation on admissible graphs:

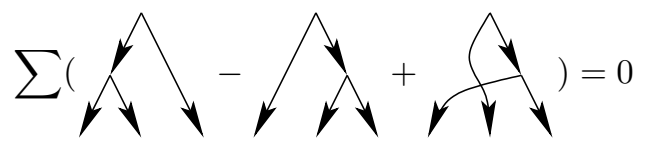

In the relations above all graphs are assumed to coincide outside the small disk shown in the figure. There may be some incoming edges entering the disk; the summation in the Jacobi relation is over all possible ways to connect these incoming edges to two depicted vertices. E.g., if there are two incoming edges, four different ways to connect these edges to two depicted vertices will give $4 \times 3=12$ terms in the Jacobi relation. For Lie-admissible graphs the situation is simpler: there may be at most one incoming edge and the unique way to connect it, so we have

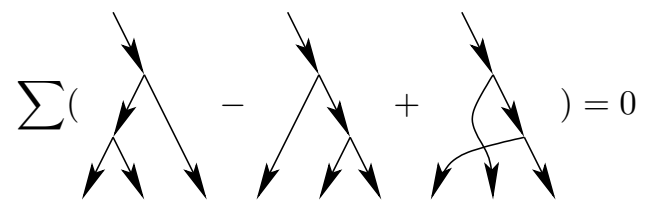

There are also obvious "labelled" versions of these relations for graphs in $\widetilde{\mathcal{G}}_{n, m}$.

Denote by $J_{n, m}$ the vector space over $\mathbb{Q}$ generated by graphs in $\mathcal{G}_{n, m}$ modulo the $A S$ and Jacobi relations, and set $J_{m}=\oplus_{n} J_{n, m}$. For $G \in \mathcal{G}_{n, m}$ denote by $[G]$ its image in $J_{n, m}$ (we will also use $[G]$ for the image of $G \in \widetilde{\mathcal{G}}_{n, m}$ under the composition of the forgetful map and the above quotient map).

Lemma 2.2. Each composition $\circ_{i}$ of graphs factors through relations (2), (3), thus defines a quotient map $\circ_{i}: J_{n, m} \times J_{n^{\prime}, m^{\prime}} \rightarrow J_{n+n^{\prime}, m+m^{\prime}-1}$

Proof. Define $[G] \circ_{i}\left[G^{\prime}\right]=\left[G \circ_{i} G^{\prime}\right]$ for any graphs $G \in \mathcal{G}_{n, m}$ and $G^{\prime} \in \mathcal{G}_{n^{\prime}, m^{\prime}}$ representing the equivalence classes $[G]$ and $\left[G^{\prime}\right]$ and extend it by linearity. The fact that $\left[\Gamma \circ_{i} G^{\prime}\right]=0$ for any linear combination $\Gamma$ such that $[\Gamma]=0$ is immediate. It is also obvious that $\left[G \circ\left(G^{\prime}-G^{\prime \prime}\right)\right]=0$ when $G^{\prime}$ and $G^{\prime \prime}$ differ by the ordering of two outgoing edges in one vertex. The only check which may cause a moment thought is that $\left[G \circ_{i} \Gamma^{\prime}\right]=0$ where $\Gamma^{\prime}$ is a linear combination of graphs appearing in 
the left hand side of equation (3). This, however, readily follows from the fact that in the definition of $o_{i}$ we sum over all possible attachments of the incoming edges of $i$-th external vertex of $G$ to vertices of $\Gamma^{\prime}$, in particular to the vertices appearing in equation (3)).

The reason to call (2) and (3) "antisymmetry and Jacobi relations" is quite simple:

Lemma 2.3. Let $\alpha$ be a Poisson bi-vector field. Then the map $B: G \mapsto B_{G}$ factors through relations (2), (3), thus defines a quotient map $B$ of $J_{m}$ to polydifferential operators.

Proof. Clearly, (2) is the graphical version of the antisymmetry relation for $\alpha$. Equation (3) with no incoming edges is exactly a graphical version of the Jacobi identity for $\alpha$. Similarly, (3) with several incoming edges corresponds to taking higher derivatives of the Jacobi identity.

\section{Configuration SPACES AND Their Maps}

3.1. Configuration spaces $C_{G}(\bar{H})$ and $C_{G}\left(\mathbb{R}^{2}\right)$. Consider the standard upper half-plane $\mathbb{H}=\{z \in \mathbb{C} \mid \Im(z)>0\}$ as the hyperbolic plane with the Lobachevsky metric, and the real line $\mathbb{R}=\{z \in \mathbb{C} \mid \Im(z)=0\}$ (together with the point $\infty$ ) as the absolute. Denote by $\bar{H}$ the compactified space $\mathbb{H} \cup \mathbb{R}$.

For a (either labelled or not) graph $G$ with $\left|V^{i}\right|=n$ internal and $\left|V^{e}\right|=m$ external vertices, let $\widetilde{C}_{G}(\bar{H})$ be the space of embeddings $f: V^{i} \cup V^{e} \rightarrow \bar{H}$ of the vertex set of $G$, such that $f\left(V^{i}\right) \subset \mathbb{H}$, and $f\left(V^{e}\right) \subset \mathbb{R}$ preserving the order of $V^{e}$. Thus we have $\widetilde{C}_{G}(\bar{H}) \cong \mathbb{H}^{n} \times \mathbb{R}^{m} \backslash \Delta$, where $\Delta$ is the union of all diagonals. Two-dimensional group $T h(\bar{H})=\left\{z \rightarrow a z+b \mid a \in \mathbb{R}_{+}, b \in \mathbb{R}\right\}$ of translations and homotheties of $\bar{H}$ acts on $\widetilde{C}_{G}(\bar{H})$. Let $C_{G}(\bar{H})$ be the quotient space $\widetilde{C}_{G} / T h(\bar{H})$. Since both $\mathbb{H}^{n} \times \mathbb{R}^{m}$ and $T h(\bar{H})$ are orientable, so is $C_{G}(\bar{H})$. This orientation does not depend on the ordering of internal vertices of $G$ : if an ordering of two internal vertices is permuted, it leads to a permutation of two copies of $\mathbb{H}$ in $\mathbb{H}^{n}$, so does not change its orientation. Further we will use the notation $C_{G}$ for $C_{G}(\bar{H})$ when $G$ has some external vertices, i.e. $m>0$.

For a graph $G$ with no external vertices one may consider also a simpler configuration space: let $\widetilde{C}_{G}\left(\mathbb{R}^{2}\right) \cong\left(\mathbb{R}^{2}\right)^{n} \backslash \Delta$ be the space of all embeddings of the vertex set of $G$ in $\mathbb{R}^{2}$. Let $C_{G}\left(\mathbb{R}^{2}\right)$ be the quotient $\widetilde{C}_{G}\left(\mathbb{R}^{2}\right) / T h\left(\mathbb{R}^{2}\right)$ modulo the action of a 3-dimensional group $T h\left(\mathbb{R}^{2}\right)=\left\{z \rightarrow a z+b \mid a \in \mathbb{R}_{+}, b \in \mathbb{C}\right\}$ of translations and homotheties of $\mathbb{R}^{2}$. Since for $m=0$ we will mostly deal with $C_{G}\left(\mathbb{R}^{2}\right)$ and not with $C_{G}(\bar{H})$, in this case we shall use the notation $C_{G}=C_{G}\left(\mathbb{R}^{2}\right)$.

3.2. Configurations of pairs of points. Let us describe in more details (see also [4) the configuration space $C=C_{e}(\bar{H})$ of a graph $G=e$ with two internal vertices connected by one edge. Acting by $T h(\bar{H})$, we may always move the outgoing vertex $v_{1}$ to $\sqrt{-1}=(0,1)$; the incoming vertex $v_{2}$ then lies on $\mathbb{H} \backslash \sqrt{-1}$. Let us describe a compactification $\bar{C}$ of $C$. To compactify $C$ when $v_{2}$ approaches $\sqrt{-1}$, we pick some $0<r<<1$ and cut out an open disc $|z-\sqrt{-1}|<r$, its boundary circle $s$ encoding the direction in which $v_{2}$ approaches $v_{1}$. To compactify $C$ at infinity, we pick $R>>1$ and cut out the set $|z|>R$, its boundary semicircle $S$ encoding the direction in which the second point approaches infinity. Finally, to compactify $C$ when $v_{2}$ approaches $\mathbb{R}$, we add an interval $I=\{z \in \mathbb{C} \mid-R<\Re(z) \leq R, \Im(z)=0\}$. 
See Figure 2 Notice also, that the space $C_{e}\left(\mathbb{R}^{2}\right) \cong S^{1}$, and may be identified with the circle $s$ after an identification of $\mathbb{R}^{2}$ with $T_{\sqrt{-1}} \mathbb{H}$.

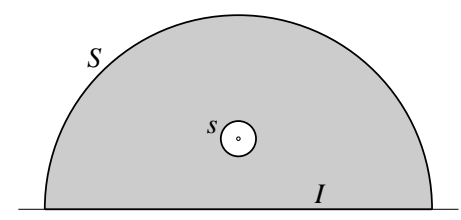

Figure 2. Compactified configuration space of pairs

Suppose now that one of the vertices of a one-edge graph is external. The corresponding configuration space is an interval; in the model above it may be identified either with the semicircle $S$ (if $v_{1}$ is external) or with the interval $I$ (if $v_{2}$ is external). Finally, suppose that both vertices are external. In this case the space $C_{e}(\bar{H})$ is a point and may be identified in the model above with one of the endpoints of the interval $I$, depending on the direction of the edge (or, equivalently, with the endpoints of the semicircle $S$ ).

Thus various boundary strata of $\bar{C}$ actually correspond to all possible configuration spaces of pairs of points.

3.3. Angle maps. The space $\bar{C}$ has the homotopy type of $S^{1}$. We will call a map $a: \bar{C} \rightarrow S^{1}$ an angle map, if it is identity on $s$, maps the whole of $S$ to 0 , and maps $I$ onto $S^{1}$ surgectively. An angle map used in 44 is defined as follows. Let $(p, q) \in \bar{H}^{2}$ be a pair of points. Compute the angle between the direction from $p$ to $\infty$ and the direction from $p$ to $q$ in the hyperbolic geometry on $\bar{H}$ (see Figure 3 ). A simple check shows that this map is $T h(\bar{H})$-invariant and indeed determines an angle map.

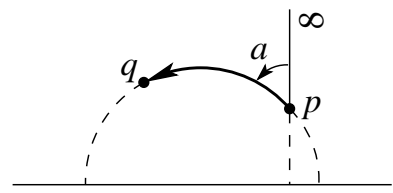

a

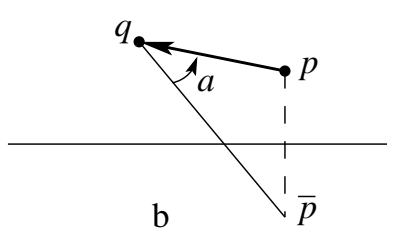

FiguRE 3. Angle maps

Here is another simple example of an angle map: for $(p, q)$ as above, take the angle between the direction from $q$ to $\bar{p}$ and from $q$ to $p$ in the Euclidean geometry on $\mathbb{C}$ (see Figure $3 \mathrm{~b}$ ).

We will fix some angle map $a$ for the remaining part of the paper.

3.4. Gauss maps and compactifications of configuration spaces. For an edge $e$ of a labelled graph $G$, an evaluation map $e v_{e}: C_{G} \rightarrow \bar{C}$ is obtained by forgetting all vertices of $G$ except for the ends of $e$. A Gauss map $\phi_{e}: C_{G} \rightarrow S^{1}$ is a composition of $e v_{e}$ with the angle map $a$. Taking the map $\phi_{e}$ for each edge $e_{1}, \ldots, e_{k}$ and using the ordering of edges, we get the product Gauss map $\Phi_{G}=$ $\prod_{e} \phi_{e}: C_{G} \rightarrow \mathbb{T}^{k}$. We shall construct compactification $\bar{C}_{G}$ of $C_{G}$ so that $\Phi_{G}$ 
will extend to $\bar{C}_{G}$. The minimal construction is to take the closure of the graph of $\phi_{G}$ in $\left(\mathbb{H}^{n} \times \mathbb{R}^{m}\right) \times \mathbb{T}^{k}$; unfortunately it is rather hard to describe explicitly. Another extreme ("overblown") solution is to blow up all diagonals of $\mathbb{H}^{n} \times \mathbb{R}^{m}$; this compactification was used in 4 . The map $\Phi_{G}$ obviously extends to any of the above compactifications.

Following [6], we will consider an intermediate solution: to blow up only the diagonals of $\mathbb{H}^{n} \times \mathbb{R}^{m}$ corresponding to subgraphs of $G$ which are 2-connected (this is somewhat smaller than in [7, where blow ups were done for all connected subgraphs). Here a graph is 2-connected (or rather vertex-2-connected), if it remains connected after a removal of any one of its vertices. In particular an edge is 2connected. The resulting space $\bar{C}_{G}$ is a manifold with corners. The boundary strata look as follows. Each codimension one face of $\bar{C}_{G}$ corresponds to a collision of all vertices of a vertex-one-connected subgraph $\Gamma$ of $G$. Both the face and the restriction of the Gauss map to it have a product structure $\Phi_{G / \Gamma} \times \Phi_{\Gamma}: C_{G / \Gamma} \times C_{\Gamma} \rightarrow$ $\mathbb{T}^{l-k} \times \mathbb{T}^{k}$, where $l$ and $k$ is the number of edges of $G$ and $\Gamma$ respectively. Here the second factor encodes the way the vertices of $\Gamma$ approach each other, seen through a "magnifying glass" (called a screen by Fulton and MacPherson). More generally, a stratum of codimension $k$ corresponds to a collection of $k$ vertex-one-connected subgraphs $\Gamma_{i}$ of $G$, each two of which are either nested (i.e. contained in one another) or disjoint. Such a stratum is adjacent to all strata of codimension $k-1$ obtained by removing one of $\Gamma_{i}$ 's from the collection.

3.5. Constructing the grand configuration space. Consider the disjoint union $\widetilde{C}_{n, m}=\amalg_{G \in \widetilde{\mathcal{G}}_{n, m}} \bar{C}_{G}$ of configuration spaces and the map $\Phi_{n, m}=\amalg_{G} \Phi_{G}: \widetilde{C}_{n, m} \rightarrow$ $\mathbb{T}^{2 n}$. The space $\widetilde{C}_{n, m}$ has no top degree cohomology since each component $\bar{C}_{G}$ has faces. We want to get rid of the faces of $\widetilde{C}_{n, m}$ by all means: gluing them together, capping-off, or relativizing, to obtain a space $C_{n, m}$ with a much smaller boundary, but still with a well-defined map $\Phi_{n, m}: C_{n, m} \rightarrow \mathbb{T}^{2 n}$.

Some boundary strata are easy to take care of; namely, we shall not care for any strata which are

- of codimension two or more in $\widetilde{C}_{n, m}$, or

- mapped to $\mathbb{T}^{2 n}$ with a loss of dimension (i.e. the difference between the dimensions of the source and the image) two or more, or

- mapped to the union $O$ of all coordinate hyperspaces $\left\{\Phi_{i}=0\right\} \subset \mathbb{T}^{2 n}$.

We will simply relativize these strata including them in a singular locus $D_{n, m}$ and considering then $\Phi_{n, m}:\left(C_{n, m}, D_{n, m}\right) \rightarrow\left(\mathbb{T}^{2 n}, O\right)$. Let us describe the gluing/relativizing process for various types of boundary faces of $\widetilde{C}_{n, m}$.

Firstly, there are the principal internal faces, where two internal vertices of a graph $G$ connected by one edge $e$ collide together. Note that the same principal face $C_{G / e} \times S^{1}$ appears in each of the three graphs related by the Jacobi relation, as shown in Figure 4 where the colliding vertices are indicated by a dashed circle (and a possible incoming edge is also dashed). Thus we can glue these faces of three graphs of Figure 4 together.

Secondly, there are the hidden internal faces $C_{G / \Gamma} \times C_{\Gamma}$, where several internal vertices of a connected subgraph $\Gamma$ of $G$ (with more than one edge) collide together. A simple dimension counting shows that unless the number of edges starting in $\Gamma$ and ending in $G \backslash \Gamma$ is two or three, the loss of dimension under the map is at least two on one of the two factors, so the face is readily relativized. Also, unless 

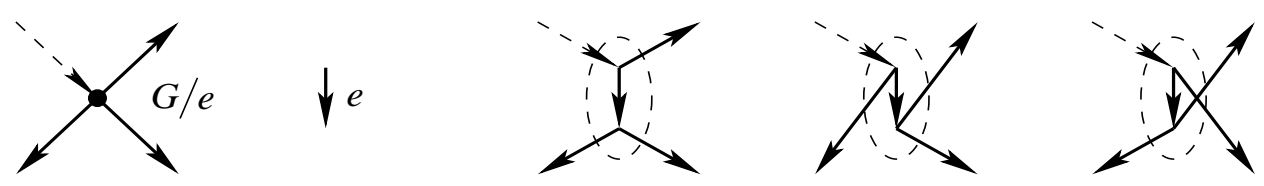

Figure 4. Gluing principal faces together

$\Gamma$ is 2-connected, $C_{\Gamma}$ loses at least one dimension under $\Phi_{G}$ (since the dilatations of one of the parts into which a cut-vertex splits $\Gamma$ preserve $\left.\Phi_{G}\right)$, so such a face can again be relativized. The remaining hidden faces ${ }^{1}$ have an orientation reversing involution which preserves $\Phi_{G}$, thus can be zipped-up (i.e. glued to itself by this involution) following an idea of []. Indeed, pick a vertex $v$ in $\Gamma$ with an edge ending in $G \backslash \Gamma$. Any vertex of a Lie-admissible graph has valence at most three; moreover, $\Gamma$ is 2-connected, thus any vertex $v \in \Gamma$ with an edge ending in $G \backslash \Gamma$ has precisely two edges $e_{1}=\left(v_{1}, v\right)$ and $e_{2}=\left(v, v_{2}\right)$ with $v_{1}, v_{2} \in \Gamma$. The involution of $v$ w.r.t. the middle of the interval connecting $v_{1}$ and $v_{2}$ (i.e. $v \rightarrow v_{1}+v_{2}-v$, if we think about $v, v_{1}$ and $v_{2}$ as points in $\mathbb{R}^{2}$ ), followed by the reordering of $e_{1}$ and $e_{2}$ reverses the orientation of this boundary face, but preserves $\Phi_{G}$.

Thirdly, there are the infinity faces, where some internal vertices of a connected subgraph $\Gamma$ of $G$ approach the absolute line $\mathbb{R}$, but remain distant from the external vertices. Then any edge starting in $\Gamma$ and ending in $G \backslash \Gamma$ is mapped to 0 , thus such faces can again be relativized.

Finally, there are the external faces, where the vertices of a connected subgraph $\Gamma$ of $G$ which collide together contain $k>0$ external vertices. Again, if there is an edge starting in $\Gamma$ and ending in $G \backslash \Gamma$, it is mapped to 0 , thus such faces can be relativized. In the remaining cases the situation depends on $k$. If $k>2$ or $k=1$, then the map $\Phi_{G / \Gamma}$ or $\Phi_{\Gamma}$ respectively has a loss of at least two dimensions, so again can be relativized. We are left, however, with the case $k=2$. We can still get rid of at least some of these faces. Namely, if the graph $G / \Gamma$ has a double edge $e_{1}=e_{2}$, we zip up a pair of faces corresponding to (a collapse of a subgraph $\Gamma$ of) two graphs $G$ and $G^{*}$ which differ only by the ordering of $e_{1}$ and $e_{2}$. Indeed, the maps $\Phi_{G}$ and $\Phi_{G^{*}}$ differ only by a transposition of two factors of $S^{1}$ in $\mathbb{T}^{2 n}$, so coincide up to a sign on the diagonal $e_{1}=e_{2}$.

To sum it up: the only boundary faces of $C_{n, m}$ (which are not included in the singular locus $D_{n, m}$ ) are the external faces, corresponding to subgraphs $\Gamma \in \mathcal{G}_{k, 2}$ of a graph $G \in \mathcal{G}_{n, m}$ which contain exactly two external and any number $k$ of internal vertices, all edges starting in $\Gamma$ also end there, and $G / \Gamma$ does not have double edges. In other words,

Proposition 3.1. [cf. Kontsevich [4] The boundary faces of $C_{n, m}$ restricted to $\bar{C}_{G}$ correspond to pairs $\Gamma^{\prime} \in \widetilde{\mathcal{G}}_{n-k, m-1}$ and $\Gamma^{\prime \prime} \in \widetilde{\mathcal{G}}_{k, 2}$ such that $G=\Gamma^{\prime} \circ_{i} \Gamma^{\prime \prime}$ for some $i$. The restriction of $\Phi_{n, m}$ to such a face is

$$
\Phi_{n-k, m-1} \times \Phi_{k, 2}: C_{\Gamma^{\prime}} \times C_{\Gamma^{\prime \prime}} \rightarrow \mathbb{T}^{2(n-k)} \times \mathbb{T}^{2 k}
$$

We will use this crucial fact in Section 3.10 below, and then again in Section 4.3

\footnotetext{
${ }^{1}$ This is actually the only place where we have to use Lie-admissibility of the initial graph, and thus the linearity of the Poisson structure
} 
3.6. Example: wedge graphs. There are two "wedge" graphs $G$ in $\mathcal{G}_{1,2}$ which differ only by the ordering of the edges; denote by $G$ the one with the natural order of the edges, and by $G^{*}$ the other. To visualize the space $C_{G}$, it is convenient to fix two external vertices in $0,1 \in \mathbb{R}$ by the action of $T h(\bar{H})$. The remaining internal vertex is free to run in the complement of these two points in $\mathbb{H}$, or, equivalently, in the complement of $0,1, \infty$ on $\bar{H}$, so $C_{G} \cong \bar{H} \backslash\{0,1, \infty\}$. The compactification $\bar{C}_{G}$ is shown on Figure 5
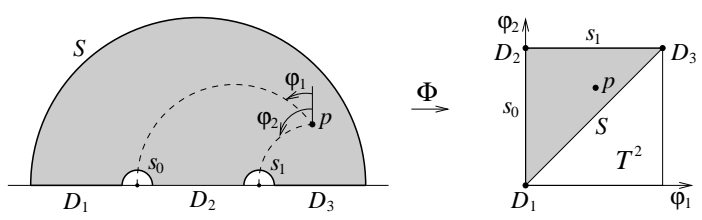

FiguRE 5. Compactified space of the wedge graph and its map to $\mathbb{T}^{2}$

The small semi-circles $s_{0}, s_{1}$ around 0,1 correspond to the faces where the internal vertex approaches one of the external vertices; $\Phi_{G}\left(s_{0}\right)=S^{1} \times\{0\}$ and $\Phi\left(s_{1}\right)=\{0\} \times S^{1}$. The singular locus $D$ is the union of the pieces $D_{1}, D_{2}, D_{3}$ of the absolute line $\mathbb{R}$ together with $s_{0}$ and $s_{1} ; \Phi_{G}(D) \subset O$ (recall that $O \subset \mathbb{T}^{2}$ is the union of the coordinate hyperspaces, i.e. $\left.O=\left\{\Phi_{1}=0\right\} \cup\left\{\Phi_{2}=0\right\}\right)$.

The large semicircle $S$ corresponds to the infinite face where two external vertices collide together (or, equivalently, the internal vertex approaches the infinity on $\mathbb{H}$ ); $\Phi_{G}(S)$ is the diagonal $\Delta \subset \mathbb{T}^{2}$. This is the only non-relativized boundary face of $\bar{C}_{G}$.

The space $\bar{C}_{G}$ is a hexagon with the boundary edges $D_{1}, s_{0}, D_{2}, s_{1}, D_{3}$ and $S$; the relativised space $\left(\bar{C}_{G}, D\right)$ is a disc with the boundary $S$. See Figure 5 .

The space $\bar{C}_{G^{*}}$ of the graph $G^{*}$ is the same; in order to keep track of the spaces we will denote the corresponding locus by $D^{*}$ and the boundary face by $S^{*}$.

Each of the open cells $C_{G}$ and $C_{G^{*}}$ has its unique 2-dimensional cohomology class, and thus the top cohomology of $C_{G} \cup C_{G^{*}}$ (or, equivalently, $H^{2}\left(\bar{C}_{G} \cup \bar{C}_{G^{*}}, D \cup\right.$ $\left.D^{*} \cup S \cup S^{*}\right)$ ) is 2-dimensional with two generators, which we may identify with $G$ and $G^{*}$.

The map $\Phi_{G^{*}}$ differs from $\Phi_{G}$ by the transposition of two copies of $S^{1}$ in $\mathbb{T}^{2}$. In particular, on the diagonal $\Delta$ of $\mathbb{T}^{2}$ both maps coincide up to a sign. Thus we may glue $\bar{C}_{G}$ to $\bar{C}_{G^{*}}$ along the remaining (non-relativised) boundary faces $S$ and $S^{*}$. The gluing however changes the cohomology: we have to add the correponding relation $G^{*}=-G$, making $H^{2}\left(C_{1,2}, D\right)$ into 1-dimensional. Indeed, $\left(C_{2,1}, D_{2,1}\right)$ is simply $S^{2}$, obtained by gluing two $\operatorname{discs}\left(\bar{C}_{G}, D\right)$ and $\left(\bar{C}_{G^{*}}, D^{*}\right)$ along their boundaries $S$ and $S^{*}$. Note, that it is mapped by $\Phi$ also to $S^{2}$, since contracting $O \subset \mathbb{T}^{2}$ into a point we get $\left(\mathbb{T}^{2}, O\right) \cong S^{2}$. See Figure [

This simple example prepares us for a general calculation of the top cohomology of the grand configuration space below.

3.7. Cohomology of the configuration space. Let us compute the top cohomology of $\bar{C}_{n, m}$. Each cell $C_{G}$ has a unique top cohomology class- the fundamental class. Thus $H^{2 n+m-2}\left(\widetilde{C}_{n, m}, X_{n, m}\right)=H^{2 n+m-2}\left(\amalg_{G} C_{G}\right)=\oplus_{G \in \widetilde{\mathcal{G}}_{n, m}} \mathbb{Q} G$, where $X_{n, m}$ is the union of all faces of $\widetilde{C}_{n, m}$. The space $C_{n, m}$ was obtained from $\widetilde{C}_{n, m}$ by 

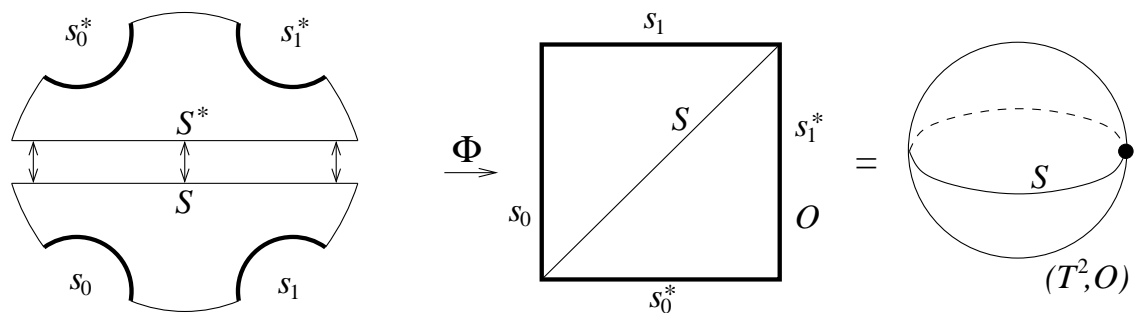

Figure 6 . Gluing $\bar{C}_{G}$ and $\bar{C}_{G^{*}}$ together and mapping them to $S^{2}$

some gluings. Each time we glue some top dimensional cells of $\widetilde{C}_{n, m}$ along a face, it adds a relation on the generators of the cohomology. The gluings along the primary internal faces were made according to the Jacobi relation. The zip-ups of the hidden strata were made according to the antisymmetry relation. No other relations were introduced during these zip-ups, since we glued together several graphs which are identical under $\Phi_{n, m}$ up to a sign; half of them are negated by the map $\Phi_{n, m}$, so the total sum is 0 . We used the antisymmetry relation again to zip up the external faces corresponding to graphs with double edges. Thus $H^{2 n+m-2}\left(C_{n, m}, \partial C_{n, m} \cup D_{n, m}\right)$ is the space of labelled Lie-admissible graphs modulo the Jacobi and the antisymmetry relations. This is not quite $J_{n, m}$, since the graphs are labelled, but not too different: forgetting a labelling gives a surjective map to $J_{n, m}$.

Thus a class in $H^{2 n+m-2}\left(C_{n, m}, \partial C_{n, m} \cup D_{n, m}\right)$ (or rather its image $Z_{n, m} \in J_{n, m}$ under the forgetful map) of a top-degree form $w$ can be written as

$$
Z_{n, m}=\sum_{G \in \widetilde{\mathcal{G}}_{n, m}} W_{G}[G]
$$

where $[G]$ is the equivalence class of $G$ in $J_{n, m}$, and the weights $W_{G}$ can be calculated as $W_{G}=\int_{C_{G}} w$. For what follows it will be important to note that if $w$ is chosen so that $\left.w\right|_{C_{G}}=d \beta$, then by the Stokes theorem we can compute the weights also as $W_{G}=\int_{\partial C_{G}} \beta$.

Remark 3.2. There is another version of this construction in which one works with unlabelled graphs, which are mapped not to $\mathbb{T}^{2 n}$, but to its quotient under the action of $S_{2 n}$ transposing the copies of $S^{1}$. While it is somewhat cleaner and requires less auxiliary data, it is harder to visualize topologically. There is, however, a simple combinatorial counterpart, which allows one to reduce the summation in (6) to unlabelled- and even further to connected- graphs, as will be explained in Section 3.8 below.

Further on we will be mostly interested in $m=2$ and $m=3$. For these values of $m$ we have $\operatorname{dim}\left(\widetilde{C}_{n, 2}\right)=2 n$, and $\operatorname{dim}\left(\widetilde{C}_{n, 3}\right)=2 n+1$, so $\widetilde{C}_{n, 2}$ is mapped by $\Phi_{n, 2}$ to the torus of the same dimension, while $\widetilde{C}_{n, 3}$ has one excessive dimension.

3.8. Two external vertices. For $m=2$ we fix a non-trivial 1-form $\omega$ on $S^{1}$ normalized by $\int_{S^{1}} \omega=1$, and take for $w$ the pull-back $\Phi_{n, m}^{*}\left(\omega^{2 n}\right)$ of the volume form $\omega^{2 n} \in \Lambda^{2 n}\left(\mathbb{T}^{2 n}\right)$. So we set

$$
W_{G}=\int_{C_{G}} \Phi_{n, m}^{*}\left(\omega^{2 n}\right)
$$


in (6). Since different labellings of a graph $G \in \mathcal{G}_{n, 2}$ lead to even transpositions of copies of $S^{1}$ in $\mathbb{T}^{2 n}$ and $\omega^{2 n}$ is invariant under such transpositions, the weight $W_{G}$ is the same for all labellings of $G$. Thus, instead of summing the weights over graphs in $\widetilde{\mathcal{G}}_{n, 2}$, we may sum over $G \in \mathcal{G}_{n, 2} / A S$ (picking any orientation and any labelling compatible with it to define $\Phi_{G}$ and $W_{G}$ ), counting each $G$ with a multiplicity being the number of its different labellings, i.e. $(2 n) ! \mid$ Aut $G \mid$, see Remark 2.1 (the additional factor of 2 disappeared since instead of counting each graph in $\mathcal{G}_{n, 2}$ twice with both orientations we take a quotent by the AS relation).

Summing over all $n$ with a formal parameter $h^{n}$ and an appropriate normalization factor, we denote by $Z$ the following generating function:

$$
Z=\sum_{n} \frac{h^{n} Z_{n, 2}}{(2 n) !}=\sum_{G \in \widetilde{\mathcal{G}}_{2}} \frac{h^{|G|} W_{G}}{(2 n) !}[G]=\sum_{G \in \mathcal{G}_{2} / A S} \frac{h^{|G|} W_{G}}{|\operatorname{Aut} G|}[G]
$$

where $|G|$ is the number of the edges of $G$. Noticing that $\Phi_{G^{\prime} \cdot G^{\prime \prime}}=\Phi_{G^{\prime}} \times \Phi_{G^{\prime \prime}}$ for any $G^{\prime}, G^{\prime \prime} \in \mathcal{G}_{2}$, we get

Lemma 3.3. The weights are multiplicative, i.e. $W_{G^{\prime} \cdot G^{\prime \prime}}=W_{G^{\prime}} W_{G^{\prime \prime}}$ for any $G^{\prime}, G^{\prime \prime} \in \mathcal{G}_{2}$.

There are still quite many terms in each degree in the definition of $Z$, since we sum over all graphs in $\mathcal{G}_{2}$. There is a standard trick of taking the logarithm, allowing one to count only connected graphs. Denote by $\mathcal{G}_{2}^{c}$ the subset of graphs in $\mathcal{G}_{2}$ which remain connected after a removal of both external vertices.

\section{Proposition 3.4.}

$$
\ln (Z)=\sum_{G \in \mathcal{G}_{2}^{c} / A S} \frac{h^{|G|} W_{G}}{|\operatorname{Aut} G|}[G]
$$

Indeed, for distinct graphs $G_{1}, \ldots, G_{k}$ we have

$$
\left|\operatorname{Aut}\left(G_{1}^{i_{1}} \cdot \ldots G_{k}^{i_{k}}\right)\right|=i_{1} ! \ldots i_{k} !\left|\operatorname{Aut} G_{1}\right|^{i_{1}} \ldots\left|\operatorname{Aut} G_{k}\right|^{i_{k}}
$$

which together with the multiplicativity of the weights proves the lemma.

3.9. Coefficients of $Z$ in small degrees. Let us check the coefficients of $Z$ for small values of $n$. The only graph in $\mathcal{G}_{0,2}$ has no internal vertices (and no edges); its weight is 1 . For $n=1$ we have two wedge graphs $G, G^{*}$, as discussed in Section 3.6. Since $\mid$ Aut $G|=|$ Aut $G^{*} \mid=1$, we get $1 / 2\left(W_{G}-W_{G^{*}}\right)[G]$ for the coefficient of $h$ in $Z$. How can we find $W_{G}-W_{G^{*}}$ without direct calculations? Note that the map $\Phi_{1,2}:\left(C_{1,2}, D_{1,2}\right) \rightarrow\left(\mathbb{T}^{2}, O\right)$ has a well-defined relative degree. It can be computed by pulling back a volume form $\omega^{2}$ on $\mathbb{T}^{2}$ and integrating it over $C_{G} \cup C_{G^{*}}$, so $\operatorname{deg}\left(\Phi_{G}\right)=\int_{C_{1,2}} \Phi_{G}^{*}\left(\omega^{2}\right)=W_{G}-W_{G^{*}}$. Another way to compute $\operatorname{deg}\left(\Phi_{G}\right)$ is to count the algebraic number of preimages $\Phi_{G}^{-1}(r)$ of any regular value $r$. Choosing some reasonable regular value, we see that there is only one preimage (e.g., for $r=(\pi / 2, \pi) \in \mathbb{T}^{2}$ we get $\left.\Phi_{G}^{-1}(r)=1+i \in C_{G}\right)$; thus the coefficient of $h$ in $Z$ does not depend on the choice of $\omega$ and is $1 / 2$.

We will use similar arguments in Section 4.2 below. 
QUANTIZATION OF LINEAR POISSON STRUCTURES AND DEGREES OF MAPS

3.10. Three external vertices. For $m=3$ the pull-back $\beta=\Phi_{n, m}^{*}\left(\omega^{2 n}\right)$ of a volume form $\omega^{2 n} \in \Lambda^{2 n}\left(\mathbb{T}^{2 n}\right)$ to $C_{n, 3}$ is a form of degree $2 n$, while $\operatorname{dim}\left(C_{n, 3}\right)=$ $2 n+1$. Note that $w=d \beta=d\left(\Phi_{n, m}^{*}\left(\omega^{2 n}\right)\right)=\Phi_{n, m}^{*}\left(d\left(\omega^{2 n}\right)\right)=0$. Thus $w$ represents the zero cohomology class in the top cohomology $H^{2 n+1}\left(C_{n, 3}, \partial C_{n, 3} \cup D\right)$. Let us now compute the same zero differently- this will give a non-trivial equation on $Z$. By Proposition 3.1 for $m=3$, each boundary face of $C_{n, 3}$ corresponds to $\Gamma^{\prime} \circ \Gamma^{\prime \prime}$ with $\Gamma^{\prime} \in \mathcal{G}_{n-k, 2}, \Gamma^{\prime \prime} \in \mathcal{G}_{k, 2}$, and the restriction of $\Phi_{n, 3}$ to it has a product structure

$$
\Phi_{\Gamma^{\prime}} \times \Phi_{\Gamma^{\prime \prime}}: C_{G} \times C_{\Gamma} \rightarrow \mathbb{T}^{2(n-k)} \times \mathbb{T}^{2 k}
$$

Therefore, as in Section 3.7 above, the image $0=w=d \beta$ in $H^{2 n+1}\left(C_{n, 3}, \partial C_{n, 3} \cup\right.$ $\left.D_{n, 3}\right)$ of $\beta \in H^{2 n}\left(\partial C_{n, 3}, D\right)$ may be calculated as

$$
\begin{aligned}
\sum_{k} \sum_{\Gamma^{\prime} \in \mathcal{G}_{n-k, 2}} \sum_{\Gamma^{\prime \prime} \in \mathcal{G}_{k, 2}} & \frac{1}{\left|\operatorname{Aut}\left(\Gamma^{\prime} \circ \Gamma^{\prime \prime}\right)\right|} \int_{C_{\Gamma^{\prime}} \times C_{\Gamma^{\prime \prime}}} \Phi_{n-k, 2}^{*}\left(\omega^{2(n-k)}\right) \times \Phi_{k, 2}^{*}\left(\omega^{2 k}\right)\left[\Gamma^{\prime} \circ \Gamma^{\prime \prime}\right] \\
& =\sum_{k}\left(\sum_{\Gamma^{\prime} \in \mathcal{G}_{n-k, 2}} \frac{W_{\Gamma^{\prime}}}{\left|\operatorname{Aut} \Gamma^{\prime}\right|}\left[\Gamma^{\prime}\right]\right) \circ\left(\sum_{\Gamma^{\prime \prime} \in \mathcal{G}_{k, 2}} \frac{W_{\Gamma^{\prime \prime}}}{\left|\operatorname{Aut} \Gamma^{\prime \prime}\right|}\left[\Gamma^{\prime \prime}\right]\right)
\end{aligned}
$$

and we conclude that

Proposition 3.5. $Z \circ Z=0$.

\section{Quantization of Poisson structures}

4.1. Constructing star products. Let $A=\Gamma\left(M, \mathcal{O}_{M}\right)$ be the algebra of $C^{\infty}$ functions on a manifold $M$. A star product is an associative $\mathbb{R}[[h]]$-linear product on $A[[h]]$ which, for functions $f, g \in A \subset A[[h]]$ is a deformation of the standard product $f g$ :

$$
f * g=f g+B_{1}(f, g) h+B_{2}(f, g) h^{2}+\ldots,
$$

where $B_{i}: A \times A \rightarrow A$ are bidifferential operators. The product then extends to $A[[h]]$ by linearity. A deformation quantization of a Poisson structure on $M$ is a construction of a star product, first term $B_{1}$ of which coincides with the (half of) Poisson bracket $\{\}:, A \times A \rightarrow A$.

Kontsevich 4 have shown that such a quantization always exists. His approach is based on an explicit construction of a star product on (an open domain of) $\mathbb{R}^{d}$, which then extends to an arbitrary $M$.

The dual of a Lie algebra has a natural Poisson structure which is linear, i.e. the components $\alpha^{i j}$ of the Poisson tensor $\alpha=\alpha^{i j} \partial_{i} \wedge \partial_{j}$ depend linearly on the coordinates $x^{i}$. Below we provide a modification of Kontsevich's original construction for linear Poisson structures.

Let $\alpha$ be a linear Poisson bi-vector field on an open domain $\mathcal{U} \subset \mathbb{R}^{d}$, defining a Poisson structure on the algebra $A$ of smooth functions on $\mathcal{U}$. Fix an arbitrary 1-form $\omega$ on $S^{1}$ and define a star product (8) using the series $Z$ of equation $\square$ and the map $B$ of Section 2.3 from graphs to bidifferential operators by

$$
f * g=B_{Z, \alpha}(f, g)
$$

for $f, g \in A$.

Returning to the computations of the coefficients of $Z$ in Section 3.9 we see that $f * g=f \cdot g+\frac{h}{2}\{f, g\}+\ldots$ Moreover, translating the statement $Z \circ Z=0$ of Proposition 3.5 to the language of differential operators, we readily obtain 
Theorem 4.1. A star product $f * g=B_{Z, \alpha}(f, g)$ is associative.

The above star product a priori depends on the initial choice of the 1-form $\omega$ on $S^{1}$. It is easy to see directly from the definition that Kontsevich's product [4] corresponds to $\omega=d \phi$ being the standard angle form on $S^{1}$.

What can be said about other 1 -forms? This is the subject of the remaining part of this note.

4.2. Digression on degrees of maps. If $C_{n, 2}$ would have no boundary faces apart from the singular locus $D_{n, 2}$, then (similarly to the case $n=1$ in Section 3.9) $Z_{n, 2}$ could be interpreted as the relative degree of a map $\Phi_{n, 2}:\left(C_{n, 2}, D_{n, 2}\right) \rightarrow\left(\mathbb{T}^{2 n}, O\right)$.

Let me clarify this point: while usually one thinks about the degree $\operatorname{deg} f$ of a map $f: M \rightarrow N$ between two manifolds being a number, one can equivalently think that it is a vector $\operatorname{deg} f[M]$ in a 1-dimensional vector space (the top cohomology of $M$, generated by the fundamental class $[M]$ of $M)$. Equivalently, $\operatorname{deg} f \operatorname{can}$ be defined as a pull-back $f^{*}([N])$ of the fundamental class of $N$, since $f^{*}([N])=$ $\operatorname{deg} f[M]$. In our setting $M$ is not a manifold, but a stratified space, so its top cohomology is not 1-dimensional, but is generated by graphs. While one can not any more define the degree as a number, the definition of degree as $f^{*}([N])$ generalizes to this situation, with all usual advantages of presenting a topological value as a degree.

In particular, we would be able to make two conclusions:

- All 1-forms $\omega$ give the same star product;

- The coefficients of this star product are rational (with the denominators arising from the normalization factors $1 /(2 n) !)$.

Unfortunately, this is not the case: the above degree is not well-defined, since in general $C_{n, 2}$ has some boundary faces (so when we change $\omega$, the value of $Z$ may change due to a "flow" through these faces). However, at least some partial steps on this way may be achieved.

4.3. Comparison of star products: 0-loop part. To understand the boundary faces, note that there is an additional grading on the configuration space by the loop number of graphs. Here the loop number $\ell(G)$ of a graph $G$ is a number of connected components appearing after a removal from $G$ of both external vertices. Indeed, the gluing relations (2) and (3) preserve the loop number. Thus $C_{n, 2}$ consists of non-connected components $C_{n, 2}^{\ell}$ corresponding to graphs with $\ell(G)=\ell$. Accordingly, any star product splits into pieces enumerated by the loop number of the corresponding graphs. Gutt's star product corresponds to the 0-loop piece:

Proposition 4.2. The 0-loop part of a star product do not depend on the choice of a 1-form $\omega$ on $S^{1}$ and coincides with the standard Gutt product.

Proof. The first statement follows from the fact that the 0 -loop part $C_{n, 2}^{0}$ of the configuration space has no non-relativized boundary faces, so the scheme discussed in Section 4.2 above works and the 0-loop part $Z_{n, 2}^{0}$ of $Z_{n, 2}$ is, up to a normalization by $1 /(2 n)$ !, a relative degree of the restriction of $\Phi_{n, 2}$ to $\left(C_{n, 2}^{0}, D_{n, 2}^{0}\right) \rightarrow\left(\mathbb{T}^{2 n}, O\right)$. Thus the 0-loop part of all star products are equal. Now results of Kathotia [3], who verified that for the standard angle 1-form on $S^{1}$ the coefficients of tree graphs coincide with the Gutt product, imply the theorem.

One can also verify this directly, in a purely combinatorial manner, computing the coefficients by counting the algebraic number of preimages in $C_{n, 2}^{0}$ of any 
regular value $r \in \mathbb{T}^{2 n}$ (so up to normalization factor $1 /(2 n)$ ! we will get integral coefficients). Note, that this counting is to be performed with labelled graphs; indeed, to pass to unlabelled graphs in Section 3.8 we used symmetries, thus would have to deal with (not regular) values on the diagonal of $\mathbb{T}^{2 n}$. A lengthy, but rather routine calculation (using Proposition 3.4 for $\ln (Z)$ ) shows that it indeed gives Gutt's product. This is illustrated in the Example 4.4 below.

4.4. Example. Let us compute the coefficients of 0-loop graphs for $n=2$, see Figure 7 . The easiest way to perform calculations is to pick a regular value $r \in \mathbb{T}^{4}$ very close to the diagonal. Say, let us take $r=(\pi, \pi+\varepsilon, \pi+2 \varepsilon, \pi+3 \varepsilon)$. When $\varepsilon \rightarrow 0$, the value approaches the diagonal and internal vertices of graphs in the preimages tend to the infinity on $\mathbb{H}^{2}$. For small $\varepsilon$ the edges connecting these vertices to the external vertices are almost Euclidean straight lines, as illustrated in Figure $7 \mathrm{~b}$. Thus calculations may be done for simplicity in the Euclidean geometry.

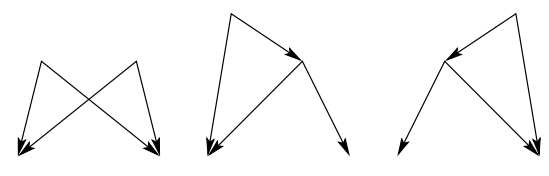

a

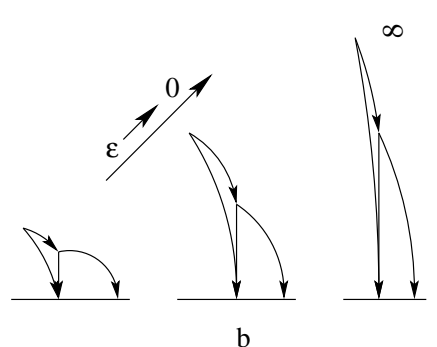

b

FiguRE 7 . Graphs in $\mathcal{G}_{2,2}$ and preimages of points approaching the diagonal

The first graph of Figure $7 \mathrm{~h}$ is the square of the wedge graph (it splits into two copies of the wedge graph after the removal of the external vertices), thus its coefficient should be $\frac{1}{2 !}\left(\frac{1}{2}\right)^{2}=\frac{1}{8}$ in view of Proposition 3.4 This is equally easy to verify diectly: out of the total of 12 different labellings of 4 edges by $\{1,2,3,4\}$ exactly 3 (namely $\left(e_{1}, e_{2}, e_{1}^{\prime}, e_{2}^{\prime}\right)=(1,2,3,4),(1,3,2,4),(1,4,2,3)$ and $(2,3,1,4)$ ) give a preimage. It is also easy to check that for all of them the local degree is +1 , so the coefficient is indeed $\frac{1}{4 !} 3=\frac{1}{8}$.

As for the second graph of Figure [7], out of the total of 24 different labellings exactly 4 give a preimage, see Figure 8 This is due to elementary geometri$\mathrm{cal} /$ combinatorial reasons. Consider the internal vertex $v$ for which one of the starting edges ends in the second internal vertex. Obviously, both edges starting in $v$ should eminate at angles which are either both smaller or both greater than the angle of the edge $e$ connecting their ends. In other words, this pair of edges should have labels either both smaller or both greater than the label of $e$; see Figure 8 It is easy to check that there are only 4 such labellings, depicted in Figure 8 . It is also easy to check that the local degree of exactly one of them (the last labelling in Figure \&) is -1 , thus the coefficient is $\frac{1}{4 !}(3-1)=\frac{1}{12}$.

By a similar inductive combinatorial arguments one can calculate the numbers of preimages of 0-loop graphs with higher $n$ (getting modified Bernulli numbers). Direct visualization of preimages becomes hard for $n>2$ since numbers of labellings (as well as denominators) grow too fast: for $n=4$ it is already 8 ! $=40320$.

4.5. Graphs with loops. It remains to study the contribution of graphs with non-zero loop numbers. For some choices of an angle form it is easy: 

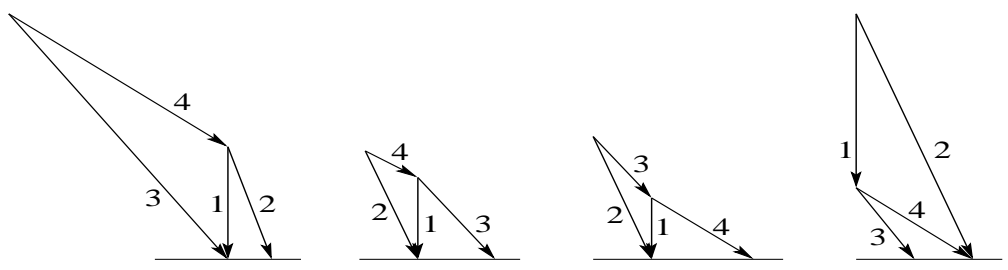

FiguRE 8. Labellings corresponding to preimages

Proposition 4.3. Let $\omega$ be any 1-form on $S^{1}$ supported on a semicircle $\{z=$ $\exp (i \psi), a<\psi<a+\pi\}$. Then the corresponding star product is the Gutt product.

Proof. Without a loss of generality, suppose that the form is supported on the lower semicircle $\{|z|=1, \Im(z)<0\}$. Now, the weight of any graph which contains an oriented cycle is zero, since such a cycle should contain an edge directed upwards. But in the linear case each graph with non-zero loop number contains such a cycle. Thus only 0-loop graphs contribute to the star product.

In other cases, the situation is more complicated and at present I fall short of computing the weights of graphs with non-zero loop numbers for arbitrary 1-forms on $S^{1}$. The remaining part of this note thus has a preliminary character. Its purpose is to explain how a computation of weights of graphs with loops (and two external vertices) can be done instead using so-called wheels, which are graphs of the type depicted in Figure 98. This gives a possibility to compute Kontsevich's weights of graphs with loops via a purely combinatorial count (see Section 4.7 below for an example).

4.6. Wheels. The above reasoning may be repeated for general $n$. Indeed, let us return to the study of the boundary faces of $C_{n, 2}$. By Proposition 3.1 these faces correspond to decompositions $G=(G / \Gamma) \circ \Gamma$ where $G / \Gamma \in \widetilde{\mathcal{G}}_{n-k, 1}$, thus is a wheel (or a product of wheels) and $\Gamma \in \widetilde{\mathcal{G}}_{k, 2}$. Let us cap-off the boundary faces by some new auxiliary configuration spaces. Recall that passing to $\ln (Z)$ instead of $Z$, we can reduce our consideration only to connected graphs; thus it would suffice to produce a $2 n$-dimensional space $\hat{C}_{G}$ with $\partial \hat{C}_{G}=C_{G}$ for each wheel $G \in \widetilde{\mathcal{G}}_{n, 1}$.

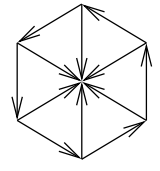

$\mathrm{a}$

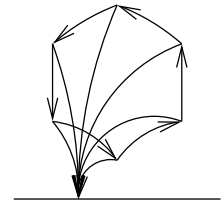

b

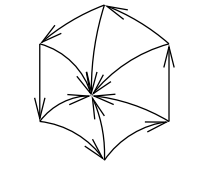

c

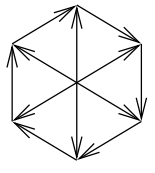

d

Figure 9. Wheels and related configuration spaces

For this purpose let us take a space of all embeddings of the vertex set of $G$ in $\bar{H}$, modulo the 2-dimensional group $T h(\bar{H})$ of translations and homotheties of $\bar{H}$, and construct its compactification $\hat{C}_{G}$ similarly to Section 3.4 This time there are no restrictions on the location of the external vertex $v$ and it is free to run over $\mathbb{H}$. Thus $\operatorname{dim}\left(\hat{C}_{G}\right)=2(n+1)-2=2 n$, and $C_{G}$ can be realized as an infinity boundary 
face of $\hat{C}_{G}$ corresponding to all embeddings for which $v \in \mathbb{R}$ and the other vertices remain in $\mathbb{H}$. An example of a wheel $G \in \widetilde{\mathcal{G}}_{6,1}$ and points in the corresponding configuration spaces $C_{G}$ and $\hat{C}_{G}$ are shown in Figures $9 \mathrm{p}, 9 \mathrm{~b}$, and 9 , respectively.

If $\hat{C}_{G}$ would have no other boundary faces, we would be done. So, let us try to get rid of all other strata of $\partial \hat{C}_{G}$. Other infinity faces are easy to get rid of: when some internal vertices approach $\mathbb{R}$ (probably together with $v$ ), some edges $e$ map under $\phi_{e}$ to 0 , so the image of $\hat{\Phi}_{G}$ belongs to $O$ and the corresponding face may be relativised. Also, we can easily get rid of almost all internal (principal and hidden) faces: when vertices of a proper subgraph $\Gamma$ of $G$ collide together, $G / \Gamma$ contains some multiple edges, so $G / \Gamma$ and hence also $G / \Gamma \circ \Gamma$ vanish due to the antisymmetry relation. But when all vertices of $G$ collide together, we get the problematic anomaly face $\hat{C}_{G}\left(\mathbb{R}^{2}\right)$, which is the space of all embeddings of the vertex set of $G$ in $\mathbb{R}^{2}$ modulo the action of $T h\left(\mathbb{R}^{2}\right)$.

There are various ways to deal with the anomaly face. One of them (not very elegant, but working) is as follows: let us consider a graph $G^{\prime}$ obtained from $G$ by the reversal of directions of all edges, see Figure $9 \mathrm{~d}$. The corresponding compactified configuration space $\hat{C}_{G^{\prime}}$ of all embeddings of the vertex set of $G^{\prime}$ in $\mathbb{H}$ is of course the same $\hat{C}_{G}$. However, the map $\hat{\Phi}_{G^{\prime}}$ differs from $\hat{\Phi}_{G}$. This time we may relativise it on all faces (including the infinite face where only $v$ approaches $\mathbb{R}$ ), except for the anomaly face corresponding to collision of all vertices. But on this anomaly face $\hat{\Phi}_{G^{\prime}}$ coincides with $\hat{\Phi}_{G}$ up to reflections of some copies of $S^{1}$ in $\mathbb{T}^{2 n}$. So, folding each copy of $S^{1}$ in two by identifying $z$ with $-z$ (or, equivalently, doubling the angle map $a \rightarrow 2 a$ ), we get the maps $2 \Phi_{G^{\prime}}$ and $2 \Phi_{G}$ to agree on the anomaly face. Thus we may glue $\bar{C}_{G^{\prime}}$ and $\bar{C}_{G}$ along the anomaly face, getting a space with the only non-relativised boundary face being the required one. This finishes the cap-off construction.

Let me illustrate an application of this idea on a simple example of graphs with $n=2$ external vertices.

4.7. Example: 2-spiked wheel. There is only one (up to a choice of an orientation) graph $\Gamma \in \mathcal{G}_{2,2}$ with $\ell(\Gamma)=1$, see Figure 10 . The only boundary face of $C_{2,2}^{1}$ which was not relativized or zipped-up corresponds to collision of the two external vertices of $\Gamma$, see Figures 10p. This face may be identified with $C_{G}$, where $G \in \mathcal{G}_{2,1}$ is a wheel with two spikes, shown in Figure 10k. We cap-off this boundary face as described above, using an auxiliary 4-dimensional configuration space $\hat{C}_{G}$, see Figure 10. The only boundary faces of $\hat{C}_{G}$ which are not mapped to the singular locus $O \in \mathbb{T}^{4}$ are $\mathbb{C}_{G}$ (correspondng to the vertex $v$ of $G$ approaching $\mathbb{R}$ ) and the anomaly face $\hat{C}_{G}\left(\mathbb{R}^{2}\right)$ (corresponding to all vertices of $G$ colliding together). In this example we can get rid of the anomaly face without using $\hat{C}_{G^{\prime}}$, simply by folding each copy of $S^{1}$ in $T^{4}$ in two (identifying $\phi$ with $\phi+\pi$ ).

After we get rid of the last boundary face $C_{G}$ of $C_{\Gamma}$ by gluing to it $\hat{C}_{G}$, we may apply the scheme of Section 4.2 to compute the relative degree of the map $\Phi_{\Gamma} \cup \hat{\Phi}_{G}:\left(C_{\Gamma} \cup \hat{C}_{G}, D\right) \rightarrow\left(\mathbb{T}^{4}, O\right)$. For any volume form $\omega$ on $\mathbb{T}^{4}$ we have

$$
\int_{C_{\Gamma}} \Phi^{*}(\omega)+\int_{\hat{C}_{G}} \hat{\Phi}^{*}(\omega)=\operatorname{deg}(\Phi \cup \hat{\Phi})
$$

We conclude that the weight $W_{\Gamma}(\omega)$ of $\Gamma$ and the weight $\hat{W}_{G}(G o)=\int_{\hat{C}_{G}} \hat{\Phi}^{*}(\omega)$ of the wheel $G$ are related by $W_{\Gamma}(\omega)+\hat{W}_{G}(\omega)=\operatorname{deg}\left(\Phi_{\Gamma} \cup \hat{\Phi}_{G}\right)$. In particular, we can 


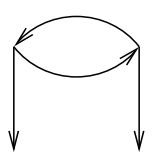

a

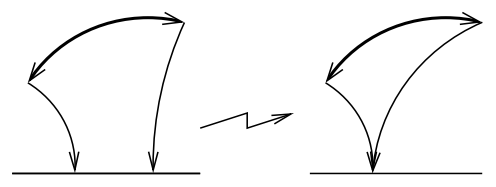

b

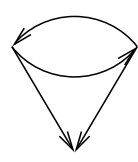

C

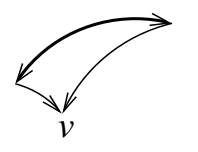

d

FIGURE 10. Graphs with two internal vertices and their embeddings

use for $\omega$ the standard uniform volume form on $\mathbb{T}^{4}$; this gives Kontsevich's weights $W_{\Gamma}^{K o}$ for $W_{\Gamma}(\omega)$ and (following Shoikhet $[$ ] $]$ ) $\hat{W}_{G}^{K o}=0$, so $W_{\Gamma}^{K o}=\operatorname{deg}\left(\Phi_{\Gamma} \cup \hat{\Phi}_{G}\right)$. On the other hand, we can compute $\operatorname{deg}\left(\Phi_{\Gamma} \cup \hat{\Phi}_{G}\right)$ counting preimages of any regular value of $\Phi_{\Gamma} \cup \hat{\Phi}_{G}$; this gives a purely combinatorial method for calculation of $W_{\Gamma}^{K o}$.

\section{REFERENCES}

[1] R. Bott, C. Taubes, On the self-linking of knots, J. Math. Phys. 35 (1994) 5247-5287

[2] S. Gutt, An explicit *-product on the cotangent bundle of a Lie group, Lett. Math. Phys. 7 (1983) 249-258

[3] V. Kathotia, Kontsevich's universal formula for deformation quantization and the CampbellBaker-Hausdorff formula, Internat. J. Math. 11 (2000) 523-551

[4] M. Kontsevich, Deformation quantization of Poisson manifolds I, q-alg/9709040 (1997)

[5] M. Kontsevich, Feynman graphs in low-dimensional topology, First European Congress in Mathematics, Vol II, Birkhäuser, 1994, 97-121

[6] G. Kuperberg, D. Thurston, Perturbative 3-manifold invariants by cut-and-paste topology, math.GT/9912167 (1999)

[7] S. Poirier, The Configuration space integral for links in $R^{3}$, Algebr. Geom. Topol. 2 (2002) $1001-1050$

[8] B. Shoikhet, Vanishing of the Kontsevich integrals of the wheels, Lett. Math. Phys. 56 (2001) 141-149

Department of Mathematics, The Technion, 32000 Haifa, Israel

E-mail address: polyak@math.technion.ac.il 